\title{
THE CENTRIFUGAL SEPARATION OF FINE MINERALS ${ }^{\star}$
}

\author{
V.M. LEPEKHIN, V.L. BORZOV AND V.N. YARAMENKO \\ Superconductivity and Solid State Physics Institute, Russian Scientific \\ Centre "Kurchatov Institute", Kurchatov Square, 123182 Moscow, Russia
}

\begin{abstract}
This paper describes a magnetofluid separator which is able to divide, with high accuracy, fine non-magnetic particles of raw materials, based on density.
\end{abstract}

(Received March 3, 1995, revised June 4, 1995)

\section{INTRODUCTION}

An important current problem in the industrial beneficiation of minerals is the treatment of ores with embedded fine particles of the useful component. The quality of ores continuously deteriorates during the course of time and it is therefore necessary to comminute as much as ore possible in order to increase the tonnage. A serious problem of reduction of the mineral particle size is closely associated with deterioration of the grade of the ore. It is obvious that the present problem tends to aggravate.

Two mutually complementing techniques, namely the gravitational beneficiation and magnetic separation, are the principal, practically universal and environmentally safe mineral processing techniques. Since it is difficult to find minerals with no difference in density or magnetic susceptibility, enrichment based on this principle can be almost always developed, even for finely dispersed fractions. The beneficiation process usually includes two stages, namely the primary concentration and the cleaning of the concentrate to obtain single mineral fraction, if possible. 
There is a number of devices for pre-treatment of fine ores (though they have numerous disadvantages) which allow to obtain high-quality concentrates. These are, for instance, the Knelson hydrostatic separator which combines the centrifugal force and reverse pressure of the inlet water [1]. Another example is a vibrating-cup separator which superimposes eccentric vibration on rotation of the $\operatorname{cup}[2]$.

The second aspect, the cleaning of the concentrate to obtain a single-mineral fraction, does not have a common solution. For instance, concentrates containing from 10 grams to several kilograms of precious metals per tonne, are known to have been stockpiled because it is not economically viable and environmentally safe to use conventional techniques for further upgrading.

Magnetic separation and separation in magnetic fluids are two techniques that can solve the above-mentioned problems, namely to divide the concentrate into the magnetic and non-magnetic fractions, and then to separate non-magnetic product according to density, with high accuracy. While there are numerous efficient laboratory magnetic separators, simple and reliable devices retaining all technological advantages of centrifugal magnetofluid separation.

Our experience in the investigation of separation of diamondiferous ores in paramagnetic fluids in strong magnetic fields generated by superconducting magnets allowed us to develop an original solution of this problem.

\section{BASIC PRINCIPLES OF CONSTRUCTION}

During the production-scale beneficiation of minerals, each component of an ore is usually removed or concentrated in one device to ensure simplicity and reliability of the operation. One-stage material separation into several fractions in a single device is performed only rarely, mainly in laboratory investigations. Taking this into account, it is our view that the following rules should be observed in the development of industrial magnetofluid (MF) separators.

1. Equipment separation raw material into two fractions should operate with high accuracy according to the "threshold concept" ("yes-no"). The first 
fraction contains particles whose density exceeds $\rho$, the other fraction contains particles with density lower than than $\rho$, where $\rho$ is the "threshold. density".

2. The separator satisfying the first condition should have a high throughput. Thus, the feed material particles must reach the threshold point as quickly as possible. This depends on the speed of rotation of the centrifugal separator and on the resistance forces. The total resistance force is the sum of the viscous drag which obeys the Stokes law, and of the buoyancy force. Moreover, in previous MF designs, the particles were overcoming the magnetic fluid buoyancy force which increased as the equilibrium was approached. Consequently, replacement of the magnetic fluid where the particle move along the threshold line, by water would accelerate the separation process.

3. Industrial separators usually operate under constant conditions for a long time so that no adjustment is needed. Therefore, a centrifugal MF separator using permanent magnets will be simpler, more economical and reliable in operation than separators with electromagnets. Adjustment of the permanent magnet separator can be performed by changing position of the magnets.

\section{DESIGN AND PERFORMANCE OF THE SEPARATOR}

Development of the centrifugal magnetofluid separator satisfying the above-mentioned conditions resulted in a model shown in Figure 1. The overall view of the separator is shown in Fig.2. The separator consists of a housing 1, rotor 2 and electric drive 3, slurry feed tube 4 and of a duct 5 for the removal of a light fraction. The rotor consists of a storage space 6 for a heavy fraction, and of a row of rectilinear permanent magnets 7 . The $\mathrm{Nd}-\mathrm{Fe}-\mathrm{B}$ permanent magnets (remanent induction $B_{\mathrm{r}}>0.95$ Tesla) have a cross-section $8 \times 4 \mathrm{~mm}$ and are placed on a circle in accordance with the magnetic field. Gap between the edges of the magnets facing the axis is $3 \mathrm{~mm}$. Non-magnetic plates 8 cover the permanent magnets. A ring splitter 9 whose inner diameter coincides with that of the permanent magnet row is situated on the upper side of the rotor. 

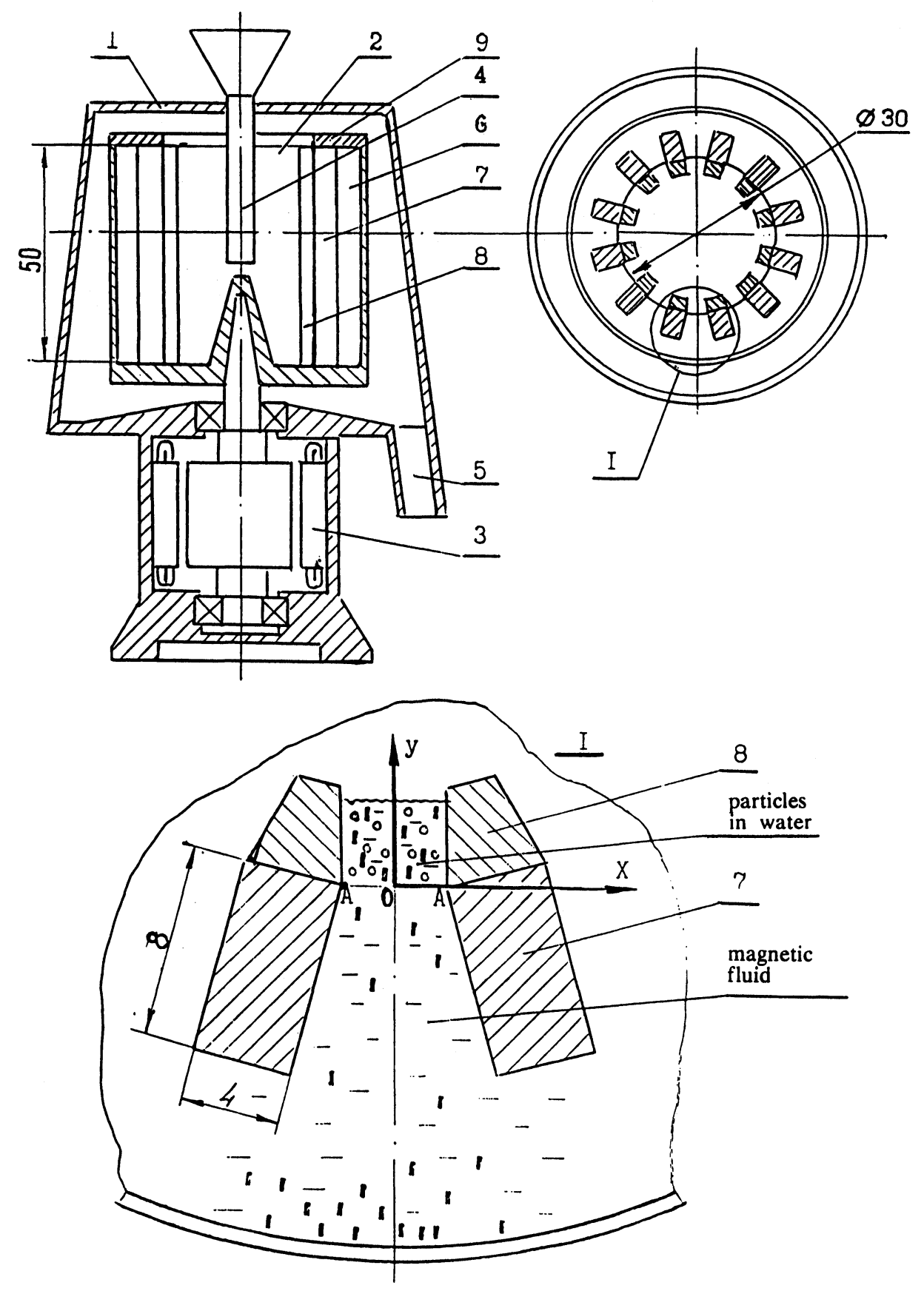

Fig.1 Schematic diagram of the centrifugal magnetofluid separator 


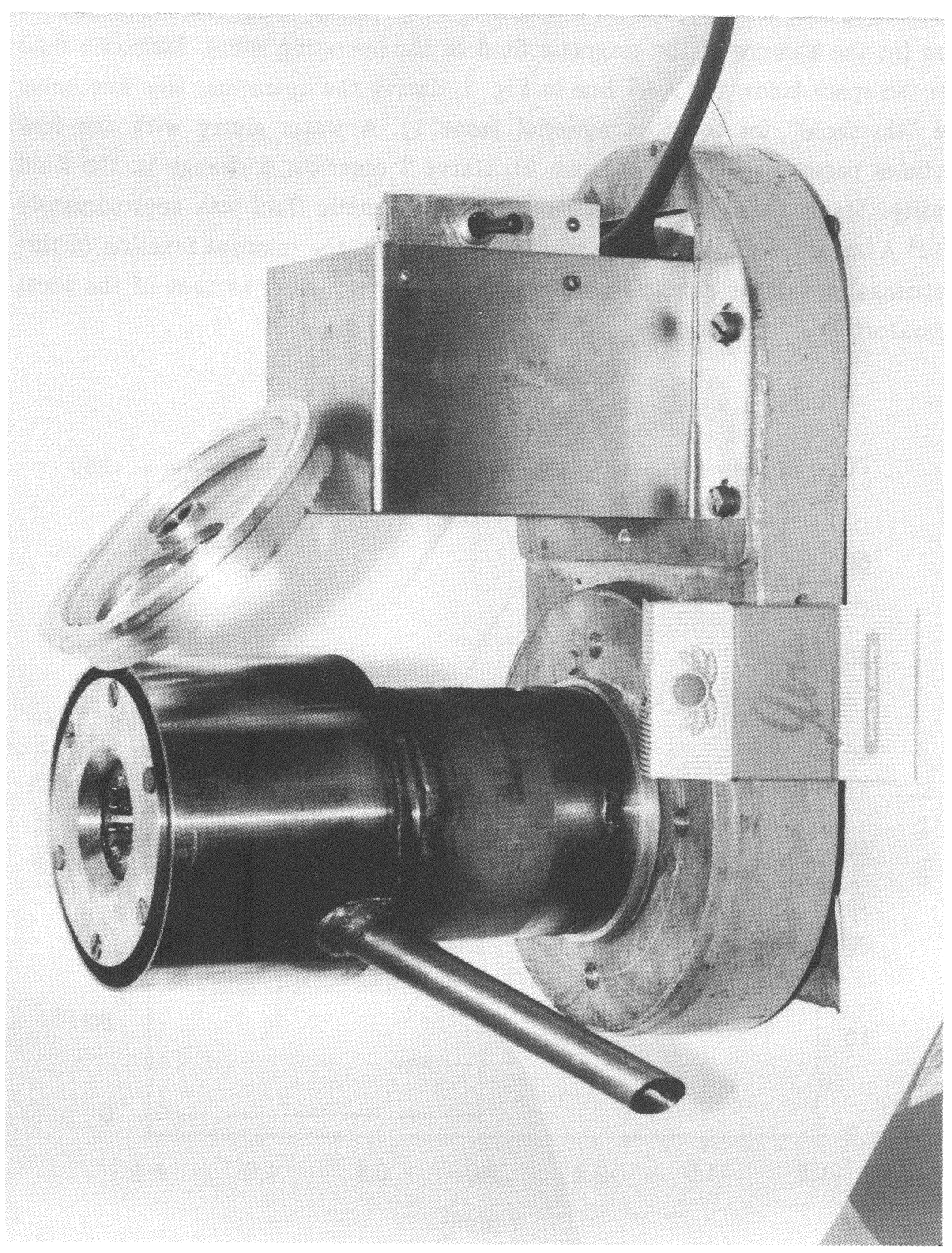

Fig. 2 The centrifugal magnetofluid separator 
Figure 3 shows a distribution of the $d B^{2} / d y$ value (curve 1) which is proportional to the magnetic force applied to a magnetic body placed along the $Y$ axis in this area (in the absence of the magnetic fluid in the operating zone). Magnetic fluid fills the space below the A-A line in Fig. 1, during the operation, this line being the "threshold" for the feed material (zone 1). A water slurry with the feed particles passes over this line (zone 2). Curve 2 describes a change in the fluid density. Magnetisation of the kerosene-based magnetic fluid was approximately $4 \times 10^{4} \mathrm{~A} / \mathrm{m}$. Curve 2 represents, practically speaking, the removal function of this centrifugal separator characteristics of which are very close to that of the ideal separator.

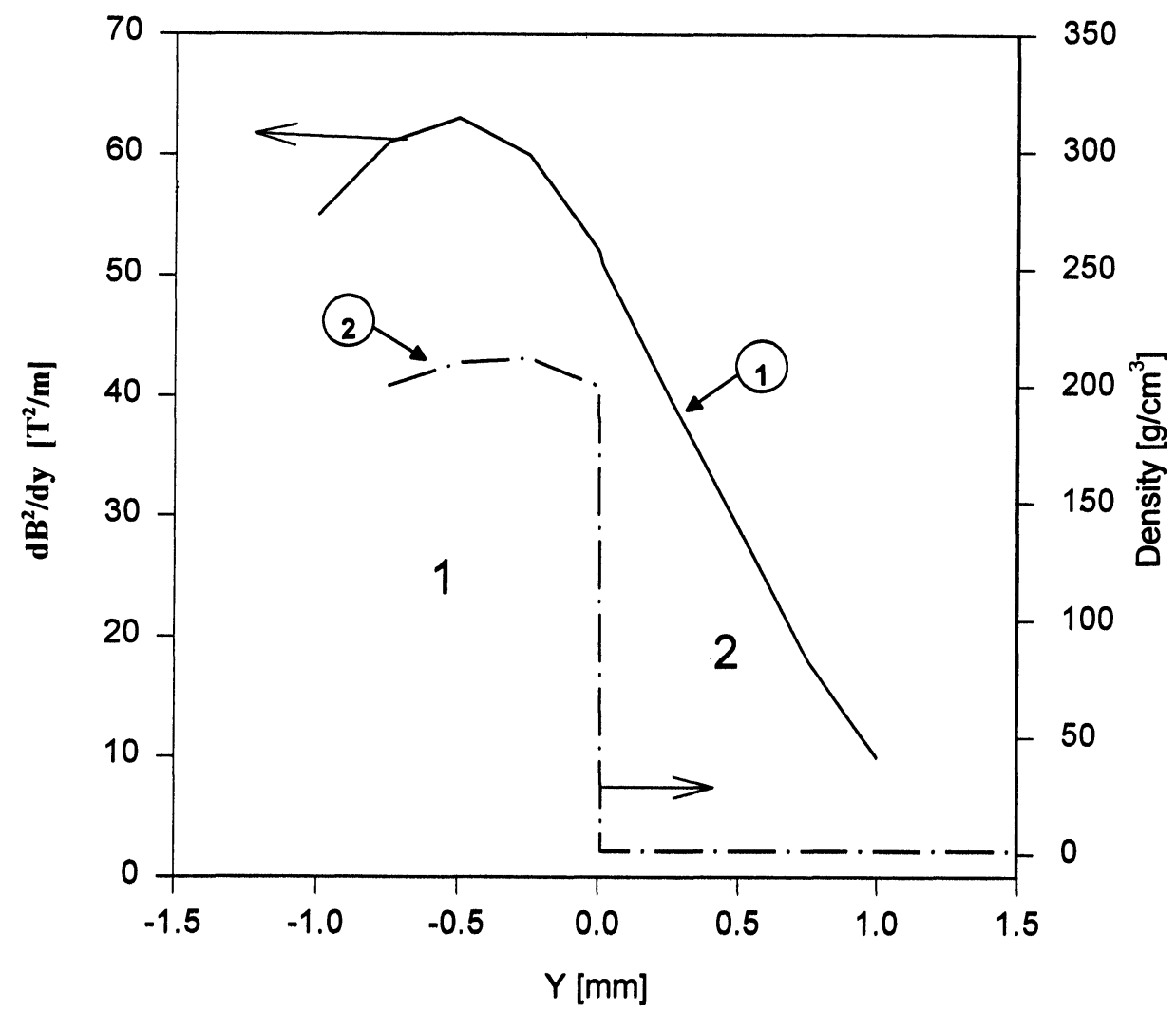

Fig. 3 Dependence of parameters along the operating zone of the MF separator: 1-curve of $\mathrm{dB}^{2} / \mathrm{dy}$, in the absence of the magnetic fluid, 2-curve of the fluid density. 
The separator operates in the following manner. At the beginning of the process, the ferromagnetic fluid is piped straight to the rotor through the slurry feed tube 4. It then fills the heavy fraction storage space, as well as gaps between permanent magnets up to the A-A level.

Thereafter, a water suspension containing the raw material is fed through the tube 4. Particles of the feed material move along the surface of the magnetic fluid from the bottom of the rotor to the discharge. These particles are transferred by the centrifugal force to the surface of the magnetic fluid, i.e. to the level threshold A-A level. Particles either overcome the buoyancy force of the magnetic fluid and settle down in the storage vessel of the heavy fraction, or flow away through the discharge tube. The heavy fraction accumulated in the storage vessel 6 must be discharged periodically.

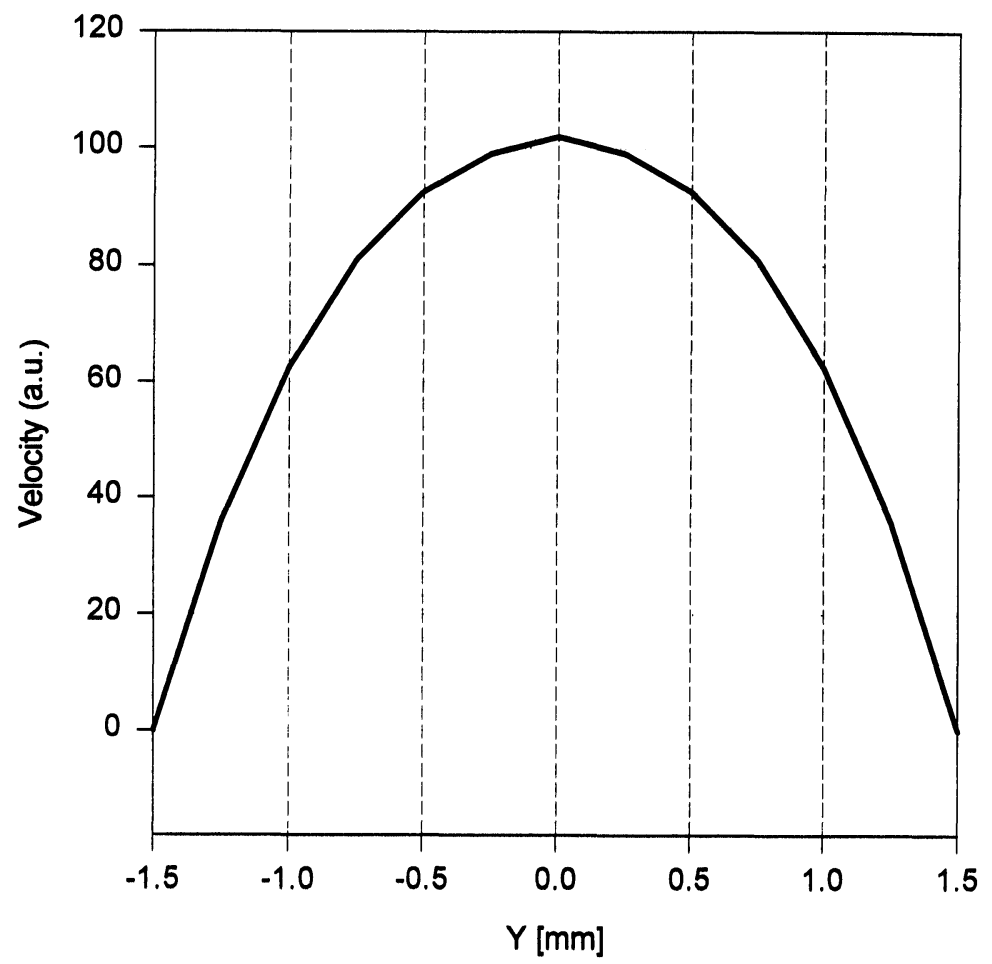

Fig. 4 The velocity distribution of of the slurry flow

In the described construction, the buoyant force of the magnetic fluid increases in the boundary zones on the threshold level. However, taking into account that the 
slurry flow through plates 8 , in laminar mode, has the velocity distribution shown in Fig. 4, the above increase does not affect the separation process significantly.

It should, however, be emphasized that if the shape of the permanent magnets corresponds to the constant buoyancy force of the threshold level, accuracy of the separation of particles according to density in the centrifugal magnetofluid separator will be higher than in the static separator. For separation of of material with density difference of $0.2 \mathrm{~g} / \mathrm{cm}^{3}$ this value will reach $10 \mathrm{~g} / \mathrm{cm}^{3}$ under the centrifugal acceleration of $50 \mathrm{~g}$ where $g$ is the acceleration of gravity. Thus, the buoyant force of the magnetic fluid can vary by as much as $10 \mathrm{~g} / \mathrm{cm}^{3}$ without affecting the removal process. This is a significant advantage of the MF centrifugal separator, together with the possibility of efficient separation of fine particles.

In the initial experiments, mixtures consisting of $-20 \mu \mathrm{m}$ quartz and copper particles were separated in the prototype separator. In subsequent experiments gold and quartz containing ore was treated. In both cases, the recovery of the useful component, namely copper or gold, exceeded $90 \%$.

\section{REFERENCES}

[1] A. Irwin: The Knelson hydrostatic concentrator. Min. Rev. 2 (4) (1982), 41

[2] P.A. Bragin: Centrifugal vibrational separator. Author's Certificate 1641425 (May 31, 1989)

Keywords: centrifugal separation, magnetofluid separator, magnetic fluid, magnetisation, permanent magnet, gold

\footnotetext{
${ }^{\star}$ A shorter version of this paper was presented at the 4th International Conference "Theoretical and Technological Problems of the Physical Processing of Raw Materials" held in Kosice, Slovakia, in October 1994.
} 